SCIREA Journal of Clinical Medicine

ISSN: 2706-8870

http://www.scirea.org/journal/CM

May 31, 2021

Volume 6, Issue 3, June 2021

\title{
The effect of early sedation depth on clinical outcomes in mechanically ventilated patients with sepsis
}

\section{Zhou-feng Wang', Heng Fan²}

${ }^{1}$ Department of Respiratory and Critical Care, Ningbo First Hospital Longshan Hospital Medical and Health Group, Ningbo, Zhejiang Province, P. R. China

${ }^{2}$ Department of Intensive Care Unit, Ningbo First Hospital, Ningbo, Zhejiang Province, P. R. China

Correspondence to: Heng Fan (Pro. PhD.), Department of Intensive care unit, Ningbo First hospital, No.59 Liuting road, Ningbo, Zhejiang province 315000, P. R. China; Tel:+86-18868655094, Fax:+86-0574-87084626, E-mail addresses: peterbenny@163.com

\begin{abstract}
:
Objective: To investigate the relationship between early sedation depth and clinical outcomes in mechanically ventilated patients with sepsis.

Methods: A prospective cohort study was conducted in an adult intensive care unit (ICU), and detailed clinical data of septic patients were collected. Multivariable analysis was used to calculate the relationship between early sedation depth and clinical outcomes, and all patients were followed up to 28 days in ICU or discharged from the hospital.
\end{abstract}

Results: A total of 100 septic patients were obtained, and 656 ICU days were followed, and 
2312 Richmond Agitation and Sedation Scale assessments were undergone. Multivariable analysis indicated that septic patients with early deep sedation had more APACHEII scores (MD: 2.78, 95\%CI: 1.18-3.97, $\mathrm{P}=0.002$ ), delirium-free days (MD: 1.12, 95\%CI: 0.41-2.01, $\mathrm{P}=0.016)$, ICU length of stay (MD: 3.31, 95\%CI: 1.76-4.54, $\mathrm{P}=0.011)$ and ventilator-free days (MD: 2.57, 95\%CI: 1.12-3.97, $\mathrm{P}=0.003$ ) than those patients with early light sedation. Kaplan-Meier analysis showed that early deep sedation was independently associated with 28-day mortality (log-rank $P=0.001)$.

Conclusion: Early light sedation may improve the clinical outcomes in mechanically ventilated patients with sepsis.

Keywords: Sedation, Sepsis, Mechanical ventilation, Mortality

\section{Introduction}

Recent studies showed that the clinical outcomes of mechanically ventilated patients with sepsis are determined by management decisions made during the initial ventilation, including the choice of sedation analgesia agents and the depth of sedation [1-3]. The short-term outcomes of septic adults are plausibly associated with the initial sedation management. To date, most sedation clinical randomized controlled trials involved patients after $48 \mathrm{~h}$ of initial mechanical ventilation, and those patients used different sedation agents and depth of sedation that lead to an unclear impact on outcomes[4,5]. Moreover, there are still no longitudinally studies to investigate early sedation choice and depth, and also strange relationship of clinical outcomes. Here, we conducted a prospective study about sedation practice in mechanically ventilated adults with sepsis, including sedation management within $48 \mathrm{~h}$ and subsequent. Our study aimed to investigate the sedation practice in ICU and clarify the relationship of early sedation depth and ventilator-free days, delirium-free days, length of ICU stay, and 28-day mortality. 


\section{Methods}

\subsection{Patient population and study design}

A prospective observational cohort study was conducted in Ningbo First Hospital in China from January 2018 to December 2019. Inclusion criteria: 1. Age more than 18-year-old, and diagnosed as sepsis [4]; 2. Mechanical ventilation continuous time was more than $48 \mathrm{~h} ; 3$. Patients had received sedative and, or analgesic medication. Exclusion criteria: 1. Age less than 18 years; 2. Psychiatric illness, neurological impairment, dementia, palliative care, and cannot communicate with investigators. All included patients were supervised by an investigator. This study was approved by the ethics committee of Ningbo First Hospital, and the informed consent were obtained from each patient before this study.

\subsection{Data collections and Definitions}

Baseline demographic data were collected, including age, weight, admission diagnosis, hospitalized process, and Acute Physiology and Chronic Health Evaluation II (APACHE II) scores. The included patients were followed from ICU admission to 28 days in ICU or discharge. The Richmond Agitation and Sedation Scale (RASS) was assessed every four hour, and the confusion assessment method for intensive care (CAM-ICU) was assessed daily by trained nurses. Dosage of sedative and analgesic medications was collected daily. Particular definitions: 1. "Early" period was defined as mechanical ventilation initiation time within $48 \mathrm{~h}$; 2. "Subsequent" period was more than $48 \mathrm{~h} ; 3$. Light sedation was a median RASS range within 48 h of -2 to +2 , deep sedation was -5 to -3 , and $\geq 3$ agitated; 4 . CAM-ICU score positive was considered as delirious.

\subsection{Statistical analysis}

Continuous data were described by medians, and interquartile ranges (IQR), means and standard deviations, and categorical variables were defined by proportions and frequencies. We used Wilcoxon rank-sum tests and Pearson chi-squared tests to test the comparisons between the deep sedation group and light sedation group. We used multiple linear regressions to calculate APACHE II scores, delirium-free days, length of ICU stay, and ventilator-free days. Kaplan-Meier survival curves with a log-rank test were created for mortality, censoring 
for 28-day ICU stay or study withdrawal. A two-sided $P$ value $<$ of 0.05 was considered to indicate statistical significance. Analysis was performed by Statistical Product and Service Solutions (SPSS 16.0, Mandalay Bay co., US).

\section{Results}

\subsection{Baseline data of the patients}

A total of 100 septic patients were enrolled, and the main came from the emergency department, 35 (35.0\%), and the general ward 65 (65.0\%) admitted after surgery. 28-day mortality of septic patients was 31 (31.0\%), ICU mortality was 30 (30\%), 3-month mortality was $39(39.0 \%)$, 6-month mortality was $44(44.0 \%)$. The baseline information of septic patients who received early deep sedation and light sedation is shown in table 1 .

Table 1. Demographics and clinical characteristics of septic patients

\begin{tabular}{cccc}
\hline Characteristics & Light sedation(n=45) & Deep sedation(n=55) & P value \\
\hline Demographic characteristics & & & \\
Age, years (IQR) & $62(45-71)$ & $53(42-61)$ & 0.074 \\
Male gender, n (\%) & $21(46.7 \%)$ & $32(58.2 \%)$ & 0.212 \\
APACHEII score, mean (SD) & $21.4 \pm 5.1$ & $21.3 \pm 4.7$ & 0.107 \\
Admission diagnosis & & & 0.111 \\
Postoperative, n (\%) & $11(24.4 \%)$ & $14(25.5 \%)$ & 0.371 \\
Respiratory failure, n (\%) & $14(31.1 \%)$ & $19(34.5 \%)$ & 0.122 \\
Renal failure, n (\%) & $12(26.7 \%)$ & $6(10.9 \%)$ & 0.193 \\
Cardiovascular, n (\%) & $7(15.6 \%)$ & $7.8 \pm 4.5$ & 0.003 \\
Clinical characteristics & & & \\
Ventilator-free days, mean (SD) & $5.1 \pm 3.6$ & & \\
\hline
\end{tabular}




\begin{tabular}{cccc} 
Hospital length of stay, mean (SD) & $13.9 \pm 6.3$ & $18.1 \pm 4.9$ & 0.001 \\
28-day mortality, n (\%) & $10(22.2 \%)$ & $21(38.2 \%)$ & 0.001 \\
ICU mortality, n (\%) & $12(26.7 \%)$ & $18(32.7 \%)$ & 0.001 \\
\hline
\end{tabular}

\subsection{Sedative and analgesic prescription}

The patients with sepsis were followed up in all of 656 ICU days. Propofol prescriptions as a sedative agent were significantly more than midazolam, given to $74(74.0 \%)$ compare to 41 $(41.0 \%)(P<0.001)$ of patients, and $151(23.0 \%)$ compare to $92(14.0 \%)$ of ICU days, respectively. Dexmedetomidine was given to 35 (35.0\%) septic patients on 89 (13.5\%) ICU days. Fentanyl and morphine as primary analgesics were used in $61(61.0 \%)$ and $8(8.0 \%)$ of septic patients, respectively.

\subsection{Sedation level}

During 656 ICU days, a total of 2312 RASS was collected. At first assessment, 55 (55.0\%) septic patients were in deep sedation (RASS: -5 to -3 ). Overall RASS assessments early, 63 patients were in deep sedation, 37 patients in light sedation (RASS: -2 to +1 ), four patients in agitated (RASS: +2 to +4 ). During the subsequent study period, 56 patients were in deep sedation, 39 patients were in light sedation, and five patients were agitated.

\subsection{Clinical outcomes}

Multivariable analysis showed that septic patients with deep sedation had a mean $(95 \% \mathrm{CI})$ of 2.78 (1.18 to 3.97) more APACHEII scores, 1.12 (0.41 to 2.01) more delirium-free days, 3.31 (1.76 to 4.54) more ICU length of stay, and 2.57 (1.12 to 3.97) more ventilator-free days than septic patients with light sedation, after adjusting for relevant covariates. Deep sedation had a more significant impact on septic patients compared with those with light sedation for APACHEII scores $(P=0.002)$, delirium-free days $(P=0.016)$, ICU length of stay $(P=0.011)$, and ventilator-free days $(P=0.003)$. Kaplan-Meier analysis indicated that septic patients with early deep sedation had a significantly higher 28-day mortality (log-rank $P=0.003)$. 


\section{Discussion}

This study provided the data showed that early sedative depth might be an essential factor in determining the clinical outcomes for patients with sepsis. The scheme of sedation together with different sedative and analgesic medications may have a more influence on the outcomes of septic patients. These results suggested that additional sedative and analgesic choices and other strategies are vitally important in those patients to suffer sepsis, and need to intervene at initial mechanical ventilation [5].

Our findings are consistent with the results of Shehabin et al. [6] study, and with strong confirmation that early deep sedation is a significant potential risk factor for increased mortality. Moreover, the results of our reasearch are neither determined by sedative regimen nor confined by the ICU model. So, these findings may be suitable to more intensive care settings, and early deep sedation may be a broader global prevalent problem related to the poor outcomes. Patients involved in our study also show similar features with patients recruited in the Pandharipande et al. [7] and interventional studies [8, 9] with a higher APACHE II score, and primary hospital diagnosis with standard ICU. As thus, we believed that our cohort study results might be suitable for similar medical conditions.

Although some studies had been reported that the sensitivity of the CAM-ICU was low [10, 11], the accuracy of delirium was evaluated as far as possible by the trained investigators in our cohort study. While sedative-related coma might be able to induce the high risk of delirium, our study also found that early deep sedation independently predict delirium. Decreasing the cases of deep sedation has been shown to improve clinical outcomes in septic patients. The schemes of reducing the sedation depth include analgo-sedation, sedation interruption, and protocolized sedation [12-14]. All the strategies which can reduce the risk of delirium trend to decrease the mortality risk. Daily sedation interruption was also common used in the practice of our cohort study. Nevertheless, multiple studies suspected whether routine sedation interruption is suitable to routinely be used in clinical practice [15].

Moreover, our study suggests that a powered care process of sedation administration is needed to solve early deep sedation problems. Early interventions are likely to settle early and 
subsequent sedation depth, and also provide safety and comfort to septic patients. The early interventions may incorporate different sedative agents to target light sedation, such as dexmedetomidine and a limited dose of propofol. Further studies should investigate the long-term mortality and cognitive function as primary outcomes in septic patients in ICUs.

Our study has several strengths and limitations. The primary force is detail, and comprehensive evaluation of involved septic patients from ICU admission to 28-day ICU stay or discharged. Then the investigators in our study were specially trained and collected the data independently, so our data quality relatively high. For the limitations, this is a prospective study in a single ICU center, and the sample size is limited and no double-blinding. So further research, we will conduct a large multicenter randomized controlled trial to clarify the relationship between the depth of sedation and the prognosis of patients with sepsis.

In Conclusion, in patients with sepsis, the early deep sedation was independently associated with more ventilator-free days and higher 28-day mortality. Our findings suggested that early light sedation may improve the clinical outcomes in septic patients. A suitable sedation and analgesia agent as a candidate for delivering interventions is supposed to be well designed within $48 \mathrm{~h}$ of initial mechanical ventilation.

\section{Acknowledgements}

This study was supported by the Cixi Agricultural and Social Development Project (CN2019035), and the Zhejiang Provincial Medical and Health Science Fund of China (2020KY815 and 2021KY998).

\section{References}

[1] van den Boogaard M, Schoonhoven L, Evers AW, et al. Delirium in critically ill patients: impact on long-term health-related quality of life and cognitive functioning. Crit Care Med, 2012;40(1):112-118.

[2] Shehabi Y, Botha JA, Boyle MS, et al. Sedation and delirium in the intensive care unit: an 
Australian and New Zealand perspective. Anaesth Intensive Care, 2008; 36:570-578.

[3] Fan H, Zhao Y, Chen GD, et al. Health insurance status and risk factors of mortality in patients with septic acute kidney injury in Ningbo, China. J Int Med Res, 2019;47(1):370-376.

[4] The American College of Chest Physicians/Society of Critical Care Medicine Consensus Conference. Definitions for sepsis and organ failure and guidelines for the use of innovative therapies in sepsis. Crit Care Med, 1992; 20:864-874.

[5] Girard TD, Kress JP, Fuchs BD, et al. Efficacy and safety of a paired sedation and ventilator weaning protocol for mechanically ventilated patients in intensive care (Awakening and Breathing Controlled trial): a randomised controlled trial. Lancet, 2008; 371:126-134.

[6] Shehabi Y, Bellomo R, Reade MC, et al. Early intensive care sedation predicts long-term mortality in mechanically ventilated critically ill patients. Am J Respir Crit Care Med, $2012 ; 186: 724-731$.

[7] Pandharipande PP, Sanders RD, Girard TD, et al. Research Effect of dexmedetomidine versus lorazepam on outcome in patients with sepsis: an a priori-designed analysis of the MENDS randomized controlled trial. Critical Care, 2010; 14(2):R38.

[8] Mehta S, Burry L, Cook D, et al. Daily sedation interruption in mechanically ventilated critically ill patients cared for with a sedation protocol: a randomized controlled trial. JAMA, 2012; 308:1985-1992.

[9] De Wit M, Gennings C, Jenvey WI, et al. Randomized trial comparing daily interruption of sedation and nursing-implemented sedation algorithm in medical intensive care unit patients. Crit Care, 2008; 12:R70.

[10] Reade MC, Eastwood GM, Peck L, et al. Routine use of the confusion assessment method for the intensive care unit (CAM-ICU) by bedside nurses may underdiagnose delirium. Crit Care Resusc, 2011; 13:217-124.

[11] Van Eijk MM, van den Boogaard M, van Marum RJ, et al. Routine use of the confusion assessment method for the intensive care unit: a multicenter study. Am J Respir Crit Care Med, 2011; 184:340-344.

[12] Augustes R, Ho KM. Meta-analysis of randomised controlled trials on daily sedation 
interruption for critically ill adult patients. Anaesth Intensive Care, 2011; 39:401-409.

[13] Fan H, Sun M, Zhu JH. Early Sedation Depth and Clinical Outcomes in Mechanically Ventilated Patients with Sepsis. Iran J Public Health, 2018;47(6):922-924.

[14] Skrobik Y, Ahern S, Leblanc M, et al. Protocolized intensive care unit management of analgesia, sedation, and delirium improves analgesia and subsyndromal delirium rates. Anesth Analg, 2010; 111:451-463.

[15] Riker RR, Shehabi Y, Bokesch PM, et al. Dexmedetomidine vs midazolam for sedation of critically ill patients: a randomized trial. JAMA, 2009; 301:489-499. 